\title{
Neurological manifestations of atrial myxoma: A retrospective analysis
}

\author{
XUE-YAN WEN $^{1 *}$, YONG-MIN CHEN $^{1 *}$, LI-LI YU $^{1}$, SHU-RONG WANG $^{1}$, HONG-BO ZHENG $^{2}$, \\ ZHI-BIN CHEN $^{1}$, LIN MA ${ }^{1}$, XIAO-PING LIAO ${ }^{1}$ and QI-FU LI ${ }^{1}$ \\ ${ }^{1}$ Department of Neurology, The First Affiliated Hospital of Hainan Medical University, Haikou, Hainan 571199; \\ ${ }^{2}$ Department of Neurology, West China Hospital, Sichuan University, Chengdu, Sichuan 610041, P.R. China
}

Received August 6, 2017; Accepted July 4, 2018

DOI: $10.3892 / \mathrm{ol} .2018 .9218$

\begin{abstract}
Atrial myxoma is the most common type of primary cardiac tumor and it is closely associated with stroke in adults. Early diagnosis and treatment of atrial myxomas is essential for the prevention of embolic events. The aim of the present study was to assess neurological complications associated with atrial myxoma. The neurological signs of atrial myxoma were retrospectively assessed in individuals who underwent treatment at West China Hospital (Chengdu, China) and The Affiliated Hospital of Hainan Medical University (Haikou, China), between March 2003 and February 2015. A total of 130 patients with atrial myxoma were included and 22 (17\%) exhibited neurologic signs. These patients were aged $39.9 \pm 12.6$ years (range, 13-78 years) and there were 13 female and 9 male patients. Ischemic cerebral infarct constituted the dominant clinical symptom $(68.2 \%)$ and 3 patients exhibited concomitant cardiac manifestations. Atrial myxoma was diagnosed by echocardiography in all patients. Irregular surface of atrial myxomas was associated with a high risk of embolic events. The patients with myxoma successfully underwent surgery with no mortality recorded. In conclusion, atrial myxomas frequently manifest as cerebral infarction in individuals without cardiovascular risk factors. These tumors more commonly affect the middle cerebral artery. Irregular surface of myxomas appears to be associated with embolic events. Echocardiography may improve the diagnosis and early treatment of atrial myxomas.
\end{abstract}

Correspondence to: Professor Qi-Fu Li or Professor Lin Ma, Department of Neurology, The First Affiliated Hospital of Hainan Medical University, 3 Xueyuan Road, Haikou, Hainan 571199, P.R. China

E-mail: lee-chief@163.com

E-mail: 13976921939@163.com

*Contributed equally

Key words: atrial myxomas, neurological complications, echocardiography, acute ischemic stroke, aneurysm

\section{Introduction}

Atrial myxomas are the most common primary cardiac tumors, comprising up to $80 \%$ of all primary tumors of the heart (1). It is widely admitted that these tumors originate from subendocardial multipotential mesenchymal cells (2). Anatomically, atrial myxomas are mostly pedunculated and soft, and which are in consistency with a smooth, villous, or irregular surface. Atrial myxomas predominantly occur between the third and the sixth decades of life and show a 2.05:1 female predominance (3). Over $75 \%$ of myxomas originate from the left atrium, $18 \%$ of them from the right atrium, while less than 5\% are found in bilateral atrium (4). Clinical manifestations of atrial myxoma vary greatly and patients usually present one of the symptoms in the following tetrad: Arrhythmias, intracardiac flow obstruction, embolic phenomena and constitutional symptoms (5). Atrial myxomas are asymptomatic in $\sim 10 \%$ of the affected individuals (6). In clinic, neurologic signs occur in $25-45 \%$ of myxoma patients, and may appear as the initial symptom in $12 \%$ individuals (7). Surgical resection is the most effective treatment and preventive intervention in atrial myxomas.

The neurological signs of atrial myxoma commonly result from ischemic cerebral infarction complications, which are found in $30-50 \%$ of patients with myxoma (8). Manifestation as haemorrhagic stroke is uncommon, and this seems to be related to the formation of cerebral aneurysms (9). The tumor type, size, location and mobility appear to be closely associated with embolism in patients with atrial myxomas (10). Cerebral infarction might recur for non-diagnosed and treated myxomas. The delayed neurological complications of myxomatous disease include oncotic aneurysms and myxomatous metastasis. They usually present with manifestations similar to cerebral vasculitis and infective endocarditis (3). Accordingly, echocardiography, computed tomography, and magnetic resonance imaging of the heart should be performed in patients with suspected stroke.

Studies of atrial myxoma and secondary brain infarction are generally small series and case reports. In this study, clinical cases of atrial myxoma-associated neurologic complications were assessed for morphology and clinical data, also summarizing their imaging characteristics. 


\section{Patients and methods}

Patients. Patient records of West China Hospital (Chengdu, China) and Affiliated Hospital of Hainan Medical University (Haikou, China) were queried for myxoma detection between March 10, 2003 and February 5, 2015, and a total of 130 patients definitely diagnosed with atrial myxoma were enrolled. After approval by the institutional review boards, 22 consecutive patients were identified with neurologic complications resulting from cardiac myxoma. The medical history of each patient was reviewed in detail, recording neurological conditions related to atrial myxoma. The patients were classified into two groups (embolic vs. non-embolic), and sex, age, cardiovascular risk factors, myxoma location and size, initial manifestation, neuroimaging assays, and clinical outcome were recorded for each of them.

Immunohistochemistry. For immunohistochemical analysis of atrium myxoma, paraffin-embedded samples were sliced and mounted on microscopic slides. Rabbit monoclonal anti-CD34 and anti-CD68 antibodies (Santa Cruz Biotechnology Inc., Dallas, TX, USA) were the first antibodies and were diluted 200 times. Antigens retrieved using citric acid buffer (10 mmol/l, pH 7.2) and by microwaving slides. The samples were treated with primary antibody at $4^{\circ} \mathrm{C}$ overnight followed by incubation with biotinylated secondary antibody (1:1,000 dilution; Zhongshan Biology Company, Beijing, China). Finally, freshly prepared DAB coloring solution was added, and CD34 or CD68 would stain brown under the microscope.

Statistical analysis. Statistical analysis was performed with SPSS 19.0 (IBM Corp., Armonk, NY, USA). Data were presented as mean \pm standard deviation (SD). Categorical variables were assessed by the chi-square test or Fisher's exact test. $\mathrm{P}<0.05$ was considered to indicate a statistically significant difference.

\section{Results}

Patient population. Neurologic complications associated with cardiac myxoma were found in 22 patients (17\%), comprising 9 male and 13 female individuals (Table I). Ages were 39.9 \pm 12.6 (range 13-78) years. Seven patients (32\%) had no remarkable medical history before diagnosis. Meanwhile, 15 subjects (68\%) showed cerebrovascular risk factors such as hyperlipidemia $(\mathrm{n}=10)$, hypertension $(\mathrm{n}=6)$, and smoking $(\mathrm{n}=5)$. Myxomas were limited to the left atrium in 20 cases (90.9\%). A total of 19 patients (86.4\%) had neurologic signs as initial presentations, while three patients initially showed cardiac signs, with neurologic symptoms occurring 2 years after surgical treatment. All patients showing neurologic complications associated with cardiac myxoma underwent neuroimaging. Brain computed tomography (CT) was performed in 19 patients (86.4\%). Twenty-one individuals (95.5\%) had brain magnetic resonance (MRI) examinations. Twelve patients $(54.5 \%)$ underwent cerebrovascular arterial imaging with magnetic resonance angiography (MRA) and computed tomography angiography (CTA). Five patients (22.7\%) were submitted to digital subtraction angiography (DSA) examinations.
Table I. Baseline clinical characteristics of patients with atrial myxoma.

\begin{tabular}{lc}
\hline Patient characteristic & $\mathrm{N}(\%)$ \\
\hline Sex & \\
Male & $9(40.9)$ \\
Female & $13(59.1)$ \\
Age, mean \pm SD & $39.9 \pm 12.6$ \\
Age range, years & $13-78$ \\
Medical conditions & \\
Hyperlipidemia & $10(45.5)$ \\
Hypertension & $6(27.3)$ \\
Smoking & $5(22.7)$ \\
Myxoma location & \\
Left atrium & $20(90.9)$ \\
Right atrium & $1(4.5)$ \\
Multichamber & $1(4.5)$ \\
Initial symptoms & \\
Dyskinesia & $12(54.5)$ \\
Speech dysfunction & $3(13.6)$ \\
Disturbance of consciousness & $2(9.1)$ \\
Headache & $1(4.5)$ \\
Vision changes & $1(4.5)$ \\
Palpitations & $2(9.1)$ \\
Dyspnoea & $1(4.5)$ \\
Neuroimaging & \\
Non-contrast CT & $19(86.4)$ \\
CTA & $2(9.1)$ \\
MRI & $21(95.5)$ \\
MRA & $12(54.5)$ \\
DSA & $5(22.7)$ \\
\hline
\end{tabular}

SD, standard deviation; CT, computed tomography; CTA, computed tomography angiography; MRI, magnetic resonance; MRA, magnetic resonance angiography; DSA, digital subtraction angiography.

Characteristics of subjects with myxoma associated cerebral infarction. The characteristics of 15 individuals developing cerebral infarction secondary to myxomatous emboli are listed in Table II. Sudden-onset unilateral motor deficit was most frequent, occurring in 13 subjects (87\%). Among the 15 cases with cerebral infarctions, 8 (53.3\%) and 7 (46.7\%), respectively, had infarcts in multiple and single vascular territories. Cardiac myxomas were surgically resected with success in all patients. The time period between the onset of neurological symptoms and resection averaged 20 days, ranging from 1 to 27 days, excluding patient 5 who suffered a transient ischemic attack (TIA) 2 years before myxoma diagnosis. Three patients (20.0\%) showed persistent neurologic deficits at final follow-up.

Neuroimaging findings. An example of combined cerebral infarction and intracranial aneurysm in a myxoma case is shown in Fig. 1. An emergency MRI of the brain demonstrated multifocal punctate acute infarction throughout the left temporal, basal ganglia and centrum semiovale (Fig. 1A). 
Table II. Characteristics of patients presenting with cerebral infarction complications of myxoma.

\begin{tabular}{|c|c|c|c|c|}
\hline Age/sex & Neurological syndrome & $\begin{array}{l}\text { Infarction location } \\
\text { on imaging }\end{array}$ & $\begin{array}{l}\text { Time elapsed between } \\
\text { cerebral infarction and } \\
\text { myxoma diagnosis }\end{array}$ & $\begin{array}{l}\text { Neurological } \\
\text { disability on last } \\
\text { follow-up }\end{array}$ \\
\hline $44 / F$ & Right-sided weakness & Left frontoparietal infarct & Simultaneous & None \\
\hline $58 / \mathrm{F}$ & $\begin{array}{l}\text { Aphasia and right-sided } \\
\text { hemiparesis }\end{array}$ & Left temporoparietal infarct & Simultaneous & $\begin{array}{l}\text { Mild persistent } \\
\text { right-sided weakness }\end{array}$ \\
\hline $22 / \mathrm{M}$ & Left-sided hemiparesis & Right frontal lobe infarct & Simultaneous & None \\
\hline $71 / \mathrm{F}$ & $\begin{array}{l}\text { Left-sided hemiparesis, } \\
\text { left arm paraesthesia }\end{array}$ & Right parietal infarct & Simultaneous & None \\
\hline $41 / \mathrm{M}$ & $\begin{array}{l}\text { Left-sided hemiparesis, } \\
\text { facial droop }\end{array}$ & $\begin{array}{l}\text { Right basal ganglia and } \\
\text { corona radiata infarcts }\end{array}$ & 2 years & None \\
\hline $67 / \mathrm{M}$ & $\begin{array}{l}\text { Visual changes, bilateral } \\
\text { Frontal headache }\end{array}$ & $\begin{array}{l}\text { Left occipital ischaemic } \\
\text { infarct }\end{array}$ & Simultaneous & $\begin{array}{l}\text { Persistent left } \\
\text { sided weakness }\end{array}$ \\
\hline $30 / \mathrm{F}$ & $\begin{array}{l}\text { Left-sided arm and leg } \\
\text { weakness }\end{array}$ & $\begin{array}{l}\text { Right parietal infarct, } \\
\text { posterior putamen and right } \\
\text { centrum semiovale }\end{array}$ & Simultaneous & None \\
\hline $50 / \mathrm{F}$ & Right arm and face weakness & $\begin{array}{l}\text { Left thalamus, midbrain and } \\
\text { temporal lobe infarct }\end{array}$ & Simultaneous & None \\
\hline $69 / \mathrm{F}$ & Left-sided arm weakness & Right parietal infarct & Simultaneous & None \\
\hline $55 / \mathrm{M}$ & Dysarthria, left hemiparesis & Multiple infarctions & Simultaneous & $\begin{array}{l}\text { Persistent right } \\
\text { sided weakness }\end{array}$ \\
\hline $23 / F$ & Right-sided weakness & Left frontoparietal infarct & Simultaneous & None \\
\hline $49 / \mathrm{M}$ & $\begin{array}{l}\text { Right facial palsy, right } \\
\text { hemiparesis }\end{array}$ & Multiple infarctions & Simultaneous & None \\
\hline $61 / F$ & Left arm weakness & $\begin{array}{l}\text { Left frontal and left occipital } \\
\text { lobe infarcts }\end{array}$ & Simultaneous & None \\
\hline $62 / \mathrm{F}$ & $\begin{array}{l}\text { Right-sided hemiparesis } \\
\text { and mixed aphasia }\end{array}$ & Left temporoparietal infarct & Simultaneous & None \\
\hline $38 / \mathrm{M}$ & Visual changes & Left occipital ischaemic infarct & Simultaneous & None \\
\hline
\end{tabular}

M, male; F, female.

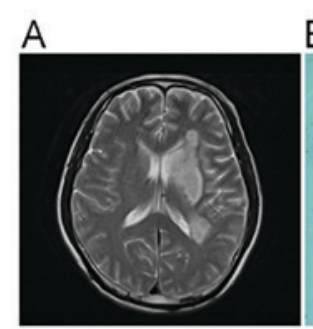

E

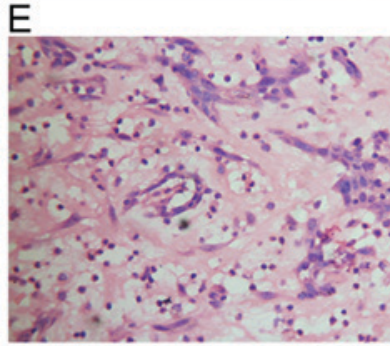

C

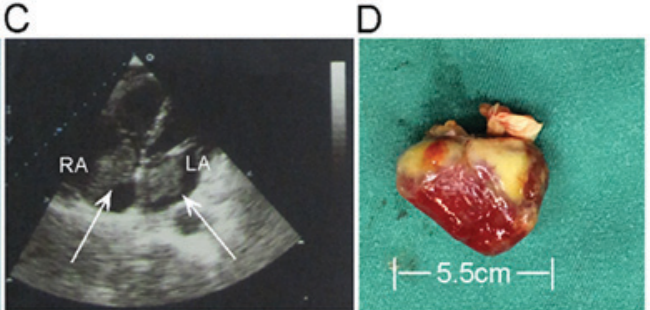

$\mathrm{F}$

G 
Table III. Atrial myxoma characteristics: Embolic vs. non-embolic groups.

\begin{tabular}{|c|c|c|c|}
\hline Myxoma characteristics & Embolic group, $\mathrm{n}=17(\%)$ & Non-embolic group, $\mathrm{n}=113(\%)$ & P-value \\
\hline Tumor diameter $(\mathrm{cm})$, mean $\pm \mathrm{SD}$ & $4.26 \pm 1.6$ & $3.18 \pm 1.1$ & 0.3720 \\
\hline$>5 \mathrm{~cm}, \mathrm{n}$ & $8(47.06)$ & $23(20.35)$ & 0.0230 \\
\hline Intratumoral haemorrhage & $10(58.82)$ & $61(53.98)$ & 0.4510 \\
\hline Attachment diameter $(\mathrm{cm})$, mean $\pm \mathrm{SD}$ & $1.0 \pm 0.2$ & $1.1 \pm 0.4$ & 0.1335 \\
\hline$>1 \mathrm{~cm}, \mathrm{n}$ & $7(41.18)$ & $51(45.13)$ & 0.4750 \\
\hline Irregular surface, $\mathrm{n}$ & $13(76.47)$ & $41(36.28)$ & 0.0273 \\
\hline
\end{tabular}

$\mathrm{n}$, number of cases; SD, standard deviation.

Digital subtraction angiography showed multiple typical distal fusiform and saccular aneurysms in left internal carotid arterial territories (Fig. 1B). Cardiac ultrasound revealed masses in the left and right atria that were resected, and identified as double atrial myxomas. The left atrium tumor and right atrium tumor measure 60x19 and 43x16 mm, respectively (Fig. 1C). The excised left atrium myxoma presents as an irregular, and measures 55x19x15 mm (Fig. 1D). Hematoxylin and eosin (H\&E) stain disclosed typical atrial myxoma cells, embedded in a loose myxoid matrix (Fig. 1E). Immunohistochemical analysis of resected atrial myxoma showed positivity for CD34 (Fig. 1F) and CD68 (Fig. 1G).

Myxoma characteristics. No significant differences were found in the diameter of myxomas between the embolic and non-embolic groups $(4.26 \pm 1.6$ vs. $3.18 \pm 1.1 \mathrm{~cm} ; \mathrm{P}=0.3720)$. However, significantly more individuals in the embolic group had large myxomas $(>5 \mathrm{~cm})$ compared with the non-embolic group (47.06 vs. $20.35 \%, \mathrm{P}=0.0230$ ). Microscopy revealed 10 myxomas with intratumoral hemorrhage $(58.82 \%)$ in the embolic group, while 61 myxomas showed intratumoral hemorrhage $(53.98 \%)$ in the non-embolic group $(\mathrm{P}=0.4510)$. Myxoma attachment was similar in both groups $(1.0 \pm 0.2 \mathrm{vs} .1 .1 \pm 0.4 \mathrm{~cm}$; $\mathrm{P}=0.1335$; Table III). However, irregular surface of myxomas was markedly more frequent in the embolic group compared with patients with no embolism (76.47 vs. $36.28 \%, \mathrm{P}=0.0273$ ).

\section{Discussion}

Atrial myxoma is found in the left atrium in $75 \%$ of patients in the solid or papillary form (2). In this study, ages (age at onset of 30-60 years) and sex distribution (female predominance) of atrial myxoma cases corroborated previous reports $(6,7)$. Neurological symptoms of atrial myxoma might appear before or at the time of primary tumor diagnosis. They were found in $16.9 \%$ of patients, a rate lower than previously reported $(4,8)$.

In this study, ischemic cerebral infarction was the most commonly encountered neurologic complication of atrial myxoma. Although embolism is a relatively common precursor of cerebral infarction in the youth, the majority of existing reports merely describe isolated cases. The present series assessed 22 patients with neurological manifestations associated with myxoma, including 15 with ischemic cerebral infarction. These data differ from previous series, which showed TIA as the most frequent neurological presentation of atrial myxoma (9). Nevertheless, multiple studies found percentages of ischemic cerebral infarction similar to the present values $(4,5,10)$. This discrepancy may be associated with different pathological characteristics of atrial myxomas. This study showed that myxomas with irregular surface were more prone to embolism. Fracturing of small myxoma fragments is considered to be responsible for embolic events. Indeed, the irregular surface contributes to myxoma fragmentation and increases interactive areas, resulting in embolism $(5,11,12)$.

Additionally, oncotic aneurysms are late signs of atrial myxoma. In patients with cardiac myxoma, oncotic aneurysms are caused by metastatic emboli, which invade and disrupt the vessel wall $(13,14)$. In the current study, 1 patient presented with recurrent cerebral infarction caused by a partially thrombosed $1.7 \mathrm{~cm}$ myxomatous oncotic aneurysm. Previous studies have examined endovascular embolization, chemotherapy, and radiation in the treatment of such aneurysms, and the findings are equivocal (15-20). Therefore, the optimal treatment of myxomatous oncotic aneurysms needs to be established.

Atrial myxoma causes cerebral infarction, but can be treated. In 22 cases in this series, neurological manifestations were found as initial sign of atrial myxoma. In 14/15 patients with cerebral infarction, tumor diagnosis occurred during the hospital stay, with surgical removal within 1 month. All patients were diagnosed by echocardiography, which was used routinely after a stroke of uncertain cause. There was no recurrence of atrial myxoma in the 22 subjects that underwent surgical treatment during follow-up (6 months to 12 years). Our findings advocate for early diagnosis and surgical resection of atrial myxoma before further embolic or cardiac complications occur.

In summary, atrial myxomas frequently present with cerebral infarction in individuals without known cardiovascular risk factors. The middle cerebral artery is more commonly concerned. Irregular surface of myxomas appears to be related to embolic events. Echocardiography could improve diagnosis and early treatment of atrial myxomas.

\section{Acknowledgments}

Help and advice from all members of the Clinical Laboratory Center of the Affiliated Hospital of Hainan Medical University is acknowledged. 


\section{Funding}

The present study was funded by the National Natural Science Foundation of China (grant no. 81460184), the Health and Family Planning Commission of Hainan Province, China, Scientific Research Project (2014-07) and the Social Development Project of Science and Technology in Hainan Province (grant no. 2015SF13).

\section{Availability of data and materials}

All data generated or analyzed during this study are included in this published article.

\section{Authors' contributions}

XYW, YMC, XPL and QFL conceived and designed the study. XYW, YMC, LLY and HBZ performed the experiments. XYW, ZBC and YMC analyzed the data. LM and SRW assisted in the study design and discussed and interpreted the data. QFL and XYW wrote the manuscript. All authors read and approved the final manuscript.

\section{Ethics approval and consent to participate}

The present study was approved by the First Affiliated Hospital of Hainan Medical University's Institutional Review Board. Individual consent was waived by the committee because of the retrospective nature of the study.

\section{Patient consent for publication}

Not applicable.

\section{Competing interests}

The authors declare that they have no competing interests.

\section{References}

1. Gošev I, Paić F, Durić Z, Gošev M, Ivčević S, Jakuš FB and Biočina B: Cardiac myxoma the great imitators: Comprehensive histopathological and molecular approach. Int J Cardiol 164: 7-20, 2013.

2. Liao WH, Ramkalawan D, Liu JL, Shi W, Zee CS, Yang XS, Li GL, Li J and Wang XY: The imaging features of neurologic complications of left atrial myxomas. Eur J Radiol 84: 933-939, 2015 .

3. Thyagarajan B, Kumar MP, Patel S and Agrawal A: Extracardiac manifestations of atrial myxomas. J Saudi Heart Assoc 29: 37-43, 2017.
4. Aiello VD and de Campos FP: Cardiac Myxoma. Autopsy Case Rep 6: 5-7, 2016.

5. Yin L, Wang J, Li W, Ling X, Xue Q, Zhang Y and Wang Z: Usefulness of CHA2DS2-VASc scoring systems for predicting risk of perioperative embolism in patients of cardiac myxomas underwent surgical treatment. Sci Rep 6: 39323, 2016.

6. Rios RE, Burmeister DB and Bean EW: Complications of atrial myxoma. Am J Emerg Med 34: 2465.e1-2465.e2, 2016.

7. Perez Andreu J, Parrilla G, Arribas JM, García-Villalba B, Lucas JJ, Garcia Navarro M, Marín F, Gutierrez F and Moreno A: Neurological manifestations of cardiac myxoma: Experience in a referral hospital. Neurologia 28: 529-534, 2013.

8. He DK, Zhang YF, Liang Y, Ye X, Wang C, Kang B and Wang ZN: Risk factors for embolism in cardiac myxoma: A retrospective analysis. Med Sci Monit 21: 1146-1154, 2015.

9. Wang Z, Chen S, Zhu M, Zhang W, Zhang H, Li H, Yuan G and Zou C: Risk prediction for emboli and recurrence of primary cardiac myxomas after resection. J Cardiothorac Surg 11: 22, 2016.

10. Spartalis M, Tzatzaki E, Spartalis E, Moris D, Athanasiou A, Kyrzopoulos S, Tsiapras D, Kalogris P and Voudris V: Atrial myxoma mimicking mitral stenosis. Cardiol Res 8: 128-130, 2017.

11. Singh S, Tripathy MP, Mohanty BB and Biswas S: Sporadic multicentric right atrial and right ventricular myxoma presenting as acute pulmonary thromboembolism. Heart Views 17: 19-22, 2016.

12. Sabzi F and Faraji R: Preoperative emboli in a pregnant woman with myxoma. Iran J Med Sci 41: 345-349, 2016.

13. Ekinci EI and Donnan GA: Neurological manifestations of cardiac myxoma: A review of the literature and report of cases. Int Med J 34: 243-249, 2004.

14. Brinjikji W, Morris JM, Brown RD, Thielen KR, Wald JT, Giannini C, Cloft HJ and Wood CP: Neuroimaging findings in cardiac myxoma patients: A single-center case series of 47 patients. Cerebrovasc Dis 40: 35-44, 2015.

15. Lee SJ, Kim JH, Na CY and Oh SS: Eleven years' experience with Korean cardiac myxoma patients: Focus on embolic complications. Cerebrovasc Dis 33: 471-479, 2012.

16. Alvarez-Sabin J, Lozano M, Sastre-Garriga J, Montoyo J, Murtra M, Abilleira S and Codina A: Transient ischaemic attack: A common initial manifestation of cardiac myxomas. Eur Neurol 45: 165-170, 2001.

17. Isogai $T$, Yasunaga $H$, Matsui $H$, Tanaka $H$, Hisagi $M$ and Fushimi K: Factors affecting in-hospital mortality and likelihood of undergoing surgical resection in patients with primary cardiac tumors. J Cardiol 69: 287-292, 2017.

18. Lee TH, Huang SC, Su TM, Yang KY and Rau CS: Multiple cerebral aneurysms and brain metastasis from primary cardiac myxosarcoma: A case report and literature review. Chang Gung Med J 34: 315-319, 2011.

19. Saffie P, Riquelme F, Mura J, Urra A, Passig C, Castro Á and Illanes S: Multiple myxomatous aneurysms with bypass and clipping in a 37-year-old man. J Stroke Cerebrovasc Dis 24: e69-e71, 2015.

20. Al-Said Y, Al-Rached H, Baeesa S, Kurdi K, Zabani I and Hassan A: Emergency excision of cardiac myxoma and endovascular coiling of intracranial aneurysm after cerebral infarction. Case Rep Neurol Med 2013: 839270, 2013. 